\title{
POLÍTICA MONETÁRIA ÓTIMA NO COMBATE À INFLAÇĀO*
}

\author{
Fernando de Holanda Barbosa**
}

\section{Resumo}

Este trabalho estabelece uma política monetária ótima para combater a inflação numa economia em que o processo inflacionário tem um componente inercial. A formulação do problema leva à aplicação da teoria do controle ótimo no qual as variáveis de estado são o hiato do produto e a taxa de inflação, e a variável de controle é a taxa de crescimento da oferta de moeda. A política monetária ótima requer uma diminuição do estoque de moeda no início do programa de estabilização e que subsequentemente a taxa de crescimento da oferta de moeda seja reduzida ao longo do tempo.

\begin{abstract}
This paper establishes an optimal monetary policy to combat inflation in an economy wherein the inflationary phenomenon contains an inertial component. The formulation of the problem leads to an application of the optimal control theory in which the state variable are the output gap and the rate of inflation, and the control variable is the growth rate of the money supply. The optimal monetary policy developed in the paper requires that at the outset of the stabilization program the monctary supply be diminished and that subsequently the rate of monctary growth be gradually reduced in the course of time.
\end{abstract}

\section{Introdução.}

Este trabalho tem como objetivo estabelecer uma política monetária ótima para o combate à inflação, numa economia em que a inflação tem um componente inercial. Este componente deve-se à existência de contratos com mecanismos de indexação baseados na inflação passada. O significado dá palavra ótima aqui é de que a trajetória da quantidade de moeda é escolhida de sorte a minimizar o custo social do programa de estabilização. A formulação do problema

\footnotetext{
* Este trabalho faz parte de uma pesquisa flnanciada pelo Programa Nacional de Pesquisa Econômica (PNPE/IPEA).

**Professor da Escola de Pós-Graduação em Economia da Fundação Getúlio Vargas
}

\begin{tabular}{llll}
\hline R. de Econometria Rio de Janeiro & v. $12, \mathrm{n}$ ․ 1, p.57-80 & abril 1992
\end{tabular}


leva a uma aplicação da teoria do controle ótimo, em que as variáveis de estado são o hiato do produto e a taxa de inflação, e a variável de controle é a taxa de crescimento da quantidade de moeda.

O trabalho está organizado do seguinte modo. A seção 2 apresenta o modelo estilizado da economia, discute a especificação da função de custo social do programa de estabilização e trata de resolver analiticamente o problema de controle. A seção 3 contém dois exemplos que ilustram a aplicação da política monetária ótima, desenvolvida na seção precedente. A seção 4 apresenta as conclusões do trabalho.

\section{Custo do programa de estabilização e política monetária} ótima.

O custo social do programa de estabilização depende do hiato do produto $h$ e da taxa de inflação $\pi$ de acordo com a função

$$
C=C(h, \pi)
$$

que satisfaz as seguintes propriedades:

$$
\frac{\partial C}{\partial h} \gtreqless 0 \Longleftrightarrow h \geq 0 \text { e } \frac{\partial C}{\partial \pi}>0
$$

O custo marginal do hiato aumenta em valor absoluto quando o produto se afasta do produto potencial e o custo marginal da inflação cresce com a taxa de inflação. Supõe-se, também, que a função de custo $C$ ( ) é convexa, o que significa dizer que a sua matriz hessiana é positiva semi-definida.

Admitiremos, por simplicidade, que a função de custo é aditiva nas variáveis $h$ e $\pi$, e que ela pode ser escrita como:

$$
C=C_{1}(h)+C_{2}(\pi)=\Phi_{1} h^{2}+\Phi_{2} \pi^{2}
$$

As funções $C_{l}(h)$ e $C_{2}(\pi)$ estão representadas na Figura 1. Nesta função o custo de manter-se a economia num nível de produção diferente do produto de pleno emprego é o mesmo, quer a economia 
esteja em recessão ou superaquecida, e o custo marginal da inflação aumenta linearmente com a taxa de inflação. ${ }^{1}$

A função de custo (2) poderia ser generalizada pela função quadrática convexa:

$$
C(h, \pi)=\Phi_{11} h^{2}+2 \Phi_{12} h \pi+\Phi_{22} \pi^{2}
$$

onde a matriz $\Phi$

$$
\Phi=\left[\begin{array}{ll}
\Phi_{11} & \Phi_{12} \\
\Phi_{12} & \Phi_{22}
\end{array}\right]
$$

é positiva semidefinida. A função de custo (2) é, então, um caso particular de (3) fazendo-se $\Phi_{12}=0$.

Quando a sociedade deseja atingir uma determinada meta da taxa de inflação, diferente de zero, a função de custo pode incorporar este objetivo, bastando para isto que se faça a seguinte modificação:

$$
C(h, \pi)=\Phi_{11} h^{2}+2 \Phi_{12} h(\pi-\bar{\pi})+\Phi_{22}(\pi-\bar{\pi})^{2}
$$

onde $\pi$ é a meta da taxa de inflação. Uma interpretação alternativa para esta formulação baseia-se no fato de que um dos custos sociais da inflação resulta da inflação não-antecipada e não da inflação propriamente dita. Nesta interpretação $\bar{\pi}$ representa a taxa de inflação esperada e $\pi-\bar{\pi}$ é a taxa de inflação não-antecipada.

A economia estilizada, em que a inflação tem um componente inercial, é descrita pelas seguintes equações diferenciais:

$$
\left\{\begin{array}{l}
\dot{\pi}=a_{11} \pi+a_{12} h+b_{1} \mu \\
\dot{h}=a_{21} \pi+a_{22} h+b_{2} \mu
\end{array}\right.
$$

onde um ponto em cima de uma variável é a derivada dela com relação ao tempo (e.g. $\dot{\pi}=d \pi / d t$ ), as letras $a_{i j}$ e $b_{i}, i, j,=1,2$, representam parâmetros e $\mu$ é a taxa de crescimento da quantidade de moeda.

${ }^{1}$ Esta função de custo é uma função de perdas (loss function) quadrática que tem sido usada por vários autores na literatura econômica (veja-se, por exemplo, Theil 1964 e Turnovsky 1981). 


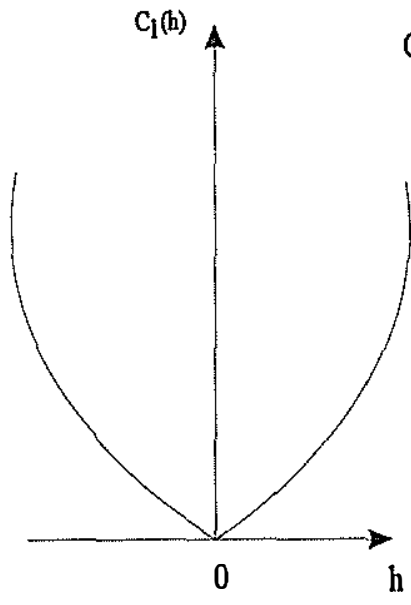

a) Custo do hiato

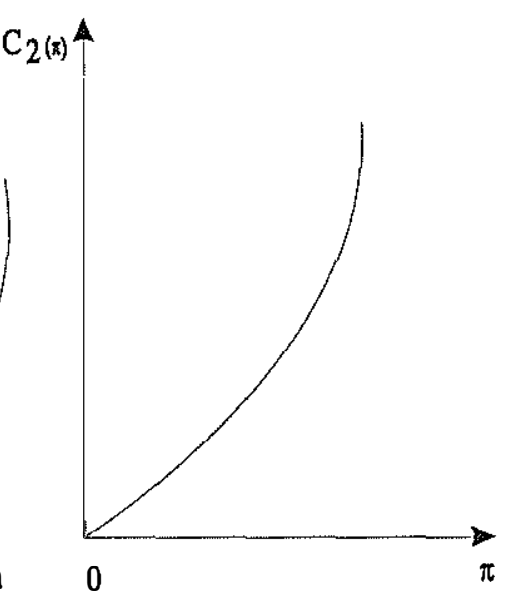

b) Custo da Inllaça

Figura 1.

Custo do Programa de Estabilização

Para que a economia seja estável, admitiremos que os elementos da matriz $A$,

$$
A=\left[\begin{array}{ll}
a_{11} & a_{12} \\
a_{21} & a_{22}
\end{array}\right]
$$

são tais que seu traço seja negativo:

$$
\operatorname{tr} A=a_{11}+a_{22}<0
$$

e que seu determinante seja positivo:

$$
|A|=a_{11} a_{22}-a_{12} a_{21}>0
$$

Admitiremos, também, que no longo prazo, quando o hiato do produto for constante e igual a zero, a taxa de inflação será constante 
.e igual à taxa de crescimento da quantidade de moeda. ${ }^{2}$ Para que isto ocorra, as seguintes restrições devem ser satisfeitas:

$$
\begin{aligned}
& b_{1}+a_{11}=0 \\
& b_{2}+a_{21}=0
\end{aligned}
$$

O problema da política monetária ótima consiste na escolha da taxa de expansão monetária $\mu$, de tal sorte que o valor atual do custo social do programa de estabilização seja minimizado, com a condição de que as equações de estado da economia sejam satisfeitas. Formalmente o problema é o seguinte:

$$
\left.\operatorname{minimizar}_{\mu} \int_{0}^{\infty} e^{-\rho t}\left(\Phi_{1} h^{2}+\Phi_{2} \pi^{2}\right)\right) d t
$$

sujeito às restrições:

$$
\left\{\begin{array}{l}
\dot{\pi}=a_{11} \pi+a_{12} h-a_{11} \mu \\
\dot{h}=a_{21} \pi+a_{22} h-a_{21} \mu
\end{array}\right.
$$

onde $\rho$ é a taxa de desconto intertemporal, que supõe-se constante ao longo do programa de estabilização.

Resta ainda para completar a especificação do problema descrever as condições iniciais da economia. Admitiremos que antes do início do programa a economia estava em pleno emprego e que a moeda vinha crescendo a uma taxa constante e igual a $\mu^{*}$, como indicado na Figura 2.

Num modelo onde a inflação tem um componente inercial, a taxa de inflação e o hiato do produto no momento $t=0$ podem mudar instantaneamente, se a política monetária for alterada, seja quanto ao estoque de moeda no instante inicial, $m(0)$, seja quanto à taxa de crescimento do estoque de moeda, $\mu(0)$. O modelo supõe que exista uma Curva de Philips do tipo

$$
\pi=\pi^{i}+\delta h
$$

${ }^{2}$ Esta hipótese supōe que a moeda é neutra no longo prazo e que, portanto, não afeta, no longo prazo, o nível do produto real da economia. 


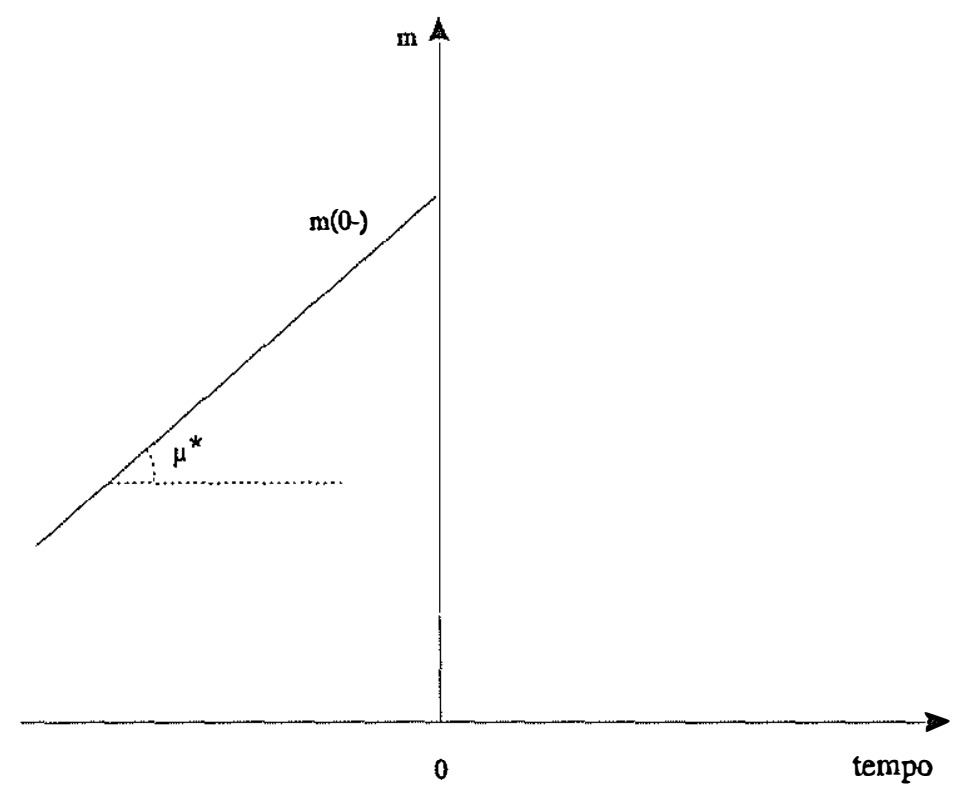

Figura 2.

Trajetória do Estoque de Moeda Antes do Programa de Estabilização

onde $\pi^{i}$ é a componente inercial e $\delta$ é um parâmetro. Se no instante inicial $\pi^{i}(0)=\pi^{*}$, os valores iniciais de $\pi(0)$ e $h(0)$ devem satisfazer:

$$
\pi(0)=\pi^{*}+\delta h(0)
$$

Outra condição que deve ser satisfeita no instante inicial é obtida eliminando-se $\mu$ das equações (3) e (4). Isto é:

$$
\dot{\pi}(0)=\frac{|A|}{a_{21}} h(0)+\frac{a_{11}}{a_{21}} \dot{h}(0)
$$

Estas duas condições iniciais, equações (7) e (8), serão úteis mais adiante na determinação do controle ótimo. Adicionalmente, iremos usar a hipótese de que a solução de controle ótimo converge para a 
solução de equilíbrio no longo prazo, $\pi=0$ e $h=0$, no problema de controle ótimo com horizonte infinito.

\subsection{Política monetária: o controle ótimo.}

A solução do problema de minimização do custo social do programa de estabilização é obtida a partir do Hamiltoniano de valor corrente $H$, definido por:

$$
\begin{aligned}
H= & \Phi_{1} h^{2}+\Phi_{2} \pi^{2}+\lambda_{1}\left(a_{11} \pi+a_{12} h-a_{11} \mu\right)+ \\
& +\lambda_{2}\left(a_{21} \pi+a_{22} h-a_{21} \mu\right)
\end{aligned}
$$

onde $\lambda_{1}$ e $\lambda_{2}$ são duas variáveis de co-estado. Rearranjando-se alguns termos, a expressão de $H$ transforma-se em:

$$
\begin{aligned}
H= & \Phi_{1} h^{2}+\Phi_{2} \pi^{2}+\left(\lambda_{1} a_{11}+\lambda_{2} a_{21}\right) \pi+\left(\lambda_{1} a_{12}+\lambda_{2} a_{22}\right) h- \\
& -\left(\lambda_{1} a_{11}-\lambda_{2} a_{22}\right) \mu
\end{aligned}
$$

O Hamiltoniano $H$ é linear na variável de controle $\mu$, a taxa de crescimento da quantidade de moeda. Se o coeficiente de $\mu$ for igual a zero, para algum intervalo de tempo, o Princípio do Máximo de Pontryagin não pode ser aplicado, pois $H$ independe do valor da variável de controle. ${ }^{3}$ Neste caso tem-se, então, um controle singular. As condições necessárias para a solução deste problema são dadas pelas seguintes equações:

$$
\begin{cases}\lambda_{1} a_{11}+\lambda_{2} a_{21} & =0 \\ \dot{\lambda}_{1} & =\rho \lambda_{1} \frac{\partial H}{\partial \pi} \\ \dot{\lambda}_{2} & =\rho \lambda_{2} \frac{\partial H}{\partial h} \\ \frac{\partial H}{\partial \lambda_{1}} & =\dot{\pi} \\ \frac{\partial H}{\partial \lambda_{2}} & =\dot{h}\end{cases}
$$

${ }^{3}$ Para exemplos de problemas de controle singular em modelos econômicos vejase, por exemplo, Clark (1976, p.88-95), Kamien e Schwartz (1981, p. 191-200) e Tu (1984, p.205-212). 
As derivadas parciais de $H$ com relação a $\pi, h, \lambda_{1}$ e $\lambda_{2}$ são iguais a:

$$
\begin{aligned}
& \frac{\partial H}{\partial \mu}=-\left(\lambda_{1} a_{11}+\lambda_{2} a_{21}\right) \\
& \frac{\partial H}{\partial \pi}=2 \Phi_{2} \pi+\left(\lambda_{1} a_{11}+\lambda_{2} a_{21}\right) \\
& \frac{\partial H}{\partial h}=2 \Phi_{1} h+\left(\lambda_{1} a_{12}+\lambda_{2} a_{22}\right) \\
& \frac{\partial H}{\partial \lambda_{1}}=a_{11} \pi+a_{12} h-a_{11} \mu \\
& \frac{\partial H}{\partial \lambda_{2}}=a_{21} \pi+a_{22} h-a_{21} \mu
\end{aligned}
$$

Substituindo-se os valores destas derivadas parciais nas equações anteriores, obtém-se:

$$
\left\{\begin{array}{l}
\lambda_{1} a_{11}+\lambda_{2} a_{21}=0 \\
\dot{\lambda}_{1}=\rho \lambda_{1}-2 \Phi_{2} \pi-\left(\lambda_{1} a_{11}+\lambda_{2} a_{21}\right. \\
\dot{\lambda}_{2}=\rho \lambda_{2}-2 \Phi_{1} h-\left(\lambda_{1} a_{12}+\lambda_{2} a_{22}\right. \\
\dot{\pi}=a_{11} \pi+a_{12} h-a_{11} \mu \\
\dot{h}=a_{21} \pi+a_{22} h-a_{21} \mu
\end{array}\right.
$$

onde a primeira equação foi repetida por conveniência. O problema consiste, então, em resolver este sistema de cinco equações nas variáveis $\lambda_{1}, \lambda_{2}, \pi, h$ e $\mu$. Para esta finalidade, adotamos o procedimento de eliminar as variáveis $\lambda_{1}$ e $\lambda_{2}$, reduzindo o sistema a três equações. Começamos combinando as equações (9) e (11) e obtendo:

$$
\dot{\lambda}_{1}=\left(\rho+\frac{a_{21} a_{12}}{a_{11}}-a_{22}\right) \lambda_{1}+2 \Phi_{1} \frac{a_{21}}{a_{11}} h
$$

Em virtude da equação (9), a equação(10) pode ser escrita como:

$$
\dot{\lambda}_{1}=\rho \lambda_{1}-2 \Phi_{2} \pi
$$


Substituindo-se esta expressão na anterior resulta:

$$
\lambda_{1}=\frac{2 \Phi_{1} a_{21}}{|A|} h+\frac{2 \Phi_{2} a_{11}}{|A|} \pi
$$

Derivando-se esta equação com relação ao tempo, tem-se:

$$
\dot{\lambda}_{1}=\frac{2 \Phi_{1} a_{21}}{|A|} \dot{h}+\frac{2 \Phi_{2} a_{11}}{|A|} \dot{\pi}
$$

Substituindo-se os valores de $\dot{\lambda}_{1}$ e $\lambda_{1}$ dados pelas equações (15) e (16) na equação (14) resulta:

$$
a_{11} \Phi_{2} \dot{\pi}+a_{21} \Phi_{1} \dot{h}=\Phi_{2}\left(a_{11} \rho-|A|\right) \pi+\Phi_{1} a_{21} \rho h
$$

Com esta equação eliminamos $\lambda_{1}$ e $\lambda_{2}$. Deve-se, agora, resolver o seguinte sistema nas variáveis $\pi, h$, e $\mu$ :

$$
\left\{\begin{array}{l}
\dot{\pi}=a_{11} \pi+a_{12} h-a_{11} \mu \\
\dot{h}=a_{21} \pi+a_{22} h-a_{21} \mu \\
a_{11} \Phi_{2} \dot{\pi}+a_{21} \Phi_{1} \dot{h}=\Phi_{2}\left(a_{11} \rho-|A|\right) \pi+\Phi_{1} a_{21} \rho h
\end{array}\right.
$$

Das equações (3a) e (4a) obtém-se:

$$
a_{21} \dot{\pi}-a_{11} \dot{h}=-|A| h
$$

O sistema de equações formado por (17a) e (18) pode ser escrito como:

$$
\left[\begin{array}{cc}
a_{11} \Phi_{2} & a_{21} \Phi_{1} \\
a_{21} & -a_{11}
\end{array}\right]\left[\begin{array}{l}
\dot{\pi} \\
\dot{h}
\end{array}\right]=\left[\begin{array}{cc}
\Phi_{2}\left(a_{11} \rho-|A|\right) & \Phi_{1} a_{21} \rho \\
0 & -|A|
\end{array}\right]\left[\begin{array}{l}
\pi \\
h
\end{array}\right]
$$

ou ainda:

$$
\left[\begin{array}{c}
\dot{\pi} \\
\dot{h}
\end{array}\right]=\left[\begin{array}{ll}
d_{11} & d_{12} \\
d_{21} & d_{22}
\end{array}\right]\left[\begin{array}{l}
\pi \\
h
\end{array}\right]
$$


onde a matriz $D=\left\{d_{i j}\right\}$ é igual a:

$$
D=\frac{1}{a_{11}^{2} \Phi_{2}+a_{21}^{2} \Phi_{1}}\left[\begin{array}{cc}
a_{11} \Phi_{2}\left(a_{11} \rho=|A|\right) & \Phi_{1} a_{21}\left(a_{11} \rho-|A|\right) \\
a_{21} \Phi_{2}\left(a_{11} \rho-|A|\right) & \Phi_{1} a_{21}^{2} \rho+a_{11} \Phi_{2}|A|
\end{array}\right]
$$

O traço desta matriz e seu determinante são:

$$
\begin{aligned}
& \operatorname{tr} D=\rho>0 \\
& |D|=\frac{\Phi_{2}\left(a_{11} \rho-|A|\right)|A|}{a_{11}^{2} \Phi_{2}+a_{21}^{2} \Phi_{1}}<0
\end{aligned}
$$

Os sinais destas desigualdades resultam do fato de que a taxa de desconto intertemporal é positiva, o traço de $A$ negativo e o determinante de $A$ é positivo.

O sistema de equações diferenciais de primeira ordem (19) produz duas equações diferenciais de segunda ordem para taxa de inflação $(x)$ e para o hiato do produto $(h)$. Elas são:

$$
\begin{aligned}
& \ddot{\pi}-(\operatorname{tr} D) \dot{\pi}+|D| \pi=0 \\
& \ddot{h}-(\operatorname{tr} D) \dot{h}+|D| h=0
\end{aligned}
$$

Como o traço da matriz $D$ é positivo e o determinante de $D$ é negativo, as duas raízes da equação característica associada à equação diferencial têm sinais contrários. Para que o sistema convirja para a solução de longo prazo escolhe-se a raiz negativa, que denominaremos por $-q$. Logo, as soluções de $\pi$ e $h$ são dadas por: ${ }^{4}$

$$
\left\{\begin{array}{l}
\pi=C_{1} e^{-q t} \\
h=C_{2} e^{-q t}
\end{array}\right.
$$

${ }^{4}$ Escolhendo-sc a raiz negativa da equação característica para a solução ótima, as seguintes condiçōes de transversalidade são satisfeitas:

$$
\lim _{t \rightarrow \infty} e^{-\rho t} \lambda_{1}(t) \pi(t)=0 \lim _{t \rightarrow \infty} e^{-\rho t} \lambda_{1}(t) h(t)=0 \lim _{t \rightarrow \infty} e^{-\rho t} \lambda_{2}(t) \pi(t)=0
$$

e

$$
\lim _{t \rightarrow \infty} e^{-\rho t} \cdot \lambda_{2}(t) \lambda(t)=0
$$


onde $C_{1}$ e $C_{2}$ são duas constantes, que dependem das condições iniciais da economia dadas pelas equações (7) e (8). Com a finalidade de analisar o que acontece no instantc inicial em que o programa de estabilização é implantado, utilizaremos um diagrama de fases, com a taxa de inflação marcada no eixo vertical e o hiato do produto no eixo horizontal, como indicado na Figura 3.

A partir do sistema de equações (19) obtém-se, com um pouco de álgebra, as seguintes equacões de $\dot{\pi}$ e $\dot{h}$ :

$$
\begin{aligned}
& \dot{\pi}=\frac{a_{11} \Phi_{2}\left(a_{11} \rho-|A|\right)}{a_{11}^{2} \Phi_{2}+a_{21}^{2} \Phi_{1}} \pi+\frac{a_{21} \Phi_{1}\left(a_{11} \rho-|A|\right)}{a_{11}^{2} \Phi_{2}+a_{21}^{2} \Phi_{1}} h \\
& \dot{h}=\frac{\Phi_{2} a_{21}\left(a_{11} \rho-|A|\right)}{a_{11}^{2} \Phi_{2}+a_{21}^{2} \Phi_{1}} \pi+\frac{a_{21}^{2} \Phi_{1} \rho+a_{11} \Phi_{2}|A|}{a_{11}^{2} \Phi_{2}+a_{21}^{2} \Phi_{1}} h
\end{aligned}
$$

O diagrama de fases da Figura 3 apresenta as combinações dos valores de $\pi$ e $h$ para os quais $\dot{\pi}=0$ e $\dot{h}=0$. As sctas indicam as trajetórias dos movimentos em cada uma das quatro regiões em que as duas retas $(\dot{\pi}=0$ e $\dot{h}=0)$ dividem o plano. O ponto onde $h=0 \mathrm{e}$ $\pi=0$ é um ponto de sela. A trajetória $S S$ converge para este ponto, enquanto a trajetória $N N$ diverge deste ponto.

Admita-se que a economia. encontra-se no momento antes do início do programa de estabilização no ponto $A$, em que a taxa de inflação é igual a $\pi(0-)$ e o hiato do produto é zero, $h(0-)=0$. A nova política monetária do programa de estabilização fará com que a economia mude inicialmente do ponto $A$ para o ponto $B$, onde o hiato do produto é negativo e a taxa de inflação é menor do que aquela que vinha ocorrendo. A economia percorrerá, então, a trajetória $S S$ convergindo para o equilíbrio de longo prazo, onde a taxa de inflação e o hiato do produto são ambos iguais a zero.

\section{Política monetária ótima: dois exemplos.}

Nesta seção apreseritaremos dois exemplos que mostram como calcular-se a política monetária ótima, determinada na seção precedente. No primeiro exemplo, a inércia inflacionária é representada analiticamente pelo mecanismo de expectativa adaptativa, que atua 


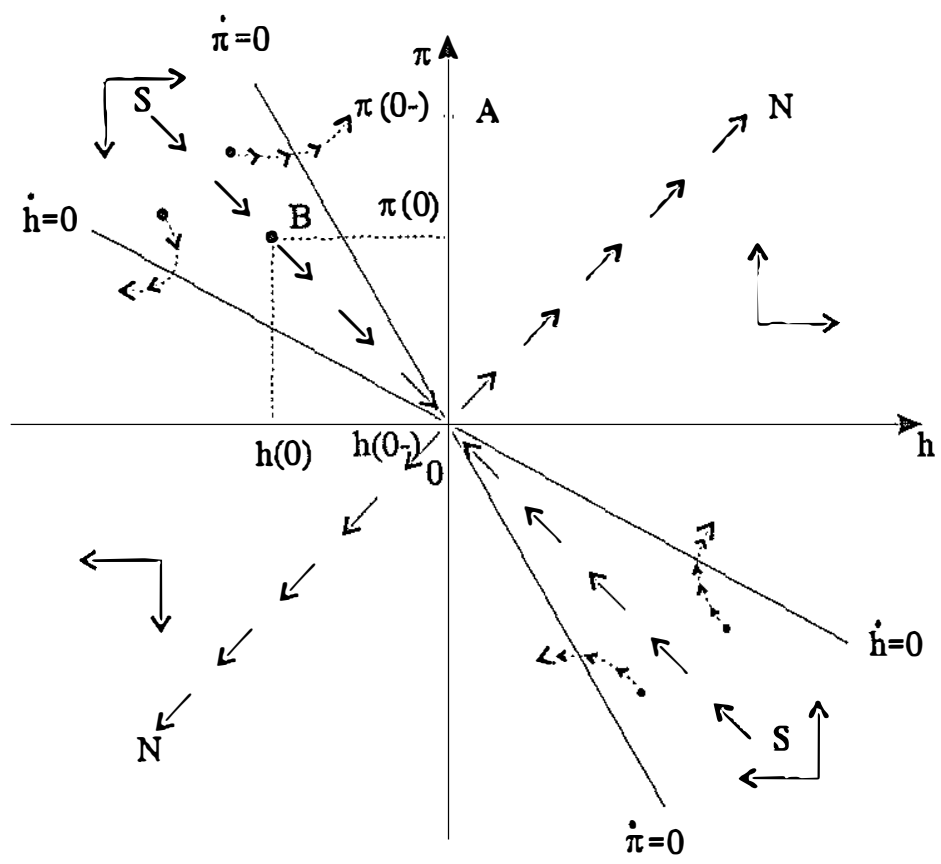

Figura 3.

Diagrama de fases

\begin{abstract}
${ }^{5}$ Uma maneira conveniente do ponto de vista analítico, para se introduzir inércia no modelo, é usar-se uma equação diferenclal do tipo $\dot{y} \theta(x-y)$. Quando se interpreta $y$ como inflação esperada e $x$ como inflação atual, a fórmula corresponde ao mecanismo de expectativa adaptativa. Mas nada impede que se chame $y$ de inflação inercial ou de "core inflation" como fazem, por exemplo Buiter e Miller (1985, p.14) para se admitir que o nível de preços e a inflação inercial, a cada instante do tempo, são variáveis predeterminadas no modelo. Esta hipótese procura captar o fato estilizado observado em economias, como a brasileira, em que a indexação é baseada em fórmulas que usam taxas de inflação observadas no passado.
\end{abstract}


tanto na demanda como na oferta agregada. ${ }^{5}$ No segundo exemplo admitiremos que na demanda agregada a previsão ć perfeita, enquanto no lado da oferta agregada existe um componente inercial na inflação que também, por conveniência analítica, será representado pela fórmula da expectativa adaptativa. ${ }^{6}$

\subsection{Modelo com expectativa adaptativa.}

Considere o modelo formado pelas seguintes equações:

$$
\begin{cases}I S: & y=c_{0}-c_{1}\left(r-\pi^{e}\right)+c_{2} f \\ L M: & m-p=a_{0}+a_{1} y-a_{2} r \\ C P: & \pi=\pi^{e}+\delta(y-\bar{y}) \\ E A: & \dot{\pi}^{e}=\theta\left(\pi-\pi^{e}\right)\end{cases}
$$

A primeira equação corresponde à curva $I S$; a segunda à curva $L M$, a terceira é a curva de Philips, e a quarta equação ć o mecanismo de expectativa adaptativa. Os símbolos têm o seguinte significado: $y$ é o (logaritmo do) produto real, $r$ é a taxa de juros nominal, $\pi^{e}$ é a taxa de inflação esperada, $f$ é uma variável de política fiscal, $m$ é o (logaritmo do) estoque de moeda, $p$ é o (logaritmo do) índice de preços, $\pi$ é a taxa de inflação $(\pi=\dot{p}=d p / d t), \bar{y}$ é o (logaritmo do) produto potencial. As letras $c_{i}, b_{i}, \delta$ e $\theta$ representam parâmetros.

A equação de demanda agregada é obtida combinando-se as curvas $I S$ e $L M$, isto é:

$$
y=k^{*}+\alpha(m-p)+\beta \pi^{e}+\gamma f
$$

\footnotetext{
${ }^{5}$ Uma maneira conveniente do ponto de vista analítico, para se introduzir inércia no modelo, é usar-se uma equação diferenclal do tipo $\dot{y} \theta(x-y)$. Quando se interpreta $y$ como inflação esperada e $x$ como inflação atual, a fórmula corresponde ao mecanismo de expectativa adaptativa. Mas nada impede que se chame $y$ de inflação inercial ou de "core inflation" como fazem, por exemplo Buiter e Miller (1985, p.14) para se admitir que o nível de preços e a inflação inercial, a cada instante do tempo, são variáveis predeterminadas no modelo. Esta hipótese procura captar o fato estilizado observado em economias, como a brasileira, em que a indexação é baseada em fórmulas que usam taxas de inflação observadas no passado.

${ }^{6}$ Esta hipótese de assimetria de expectativas no modelo tem uma longa tradição na macroeconomia moderna (veja-se, por exemplo, Dornbusch 1976). Ela supōe que os mercados financeiros olham para frente (forward looking) enquanto os mercados de mão-de-obra e de bens e serviços olham para trás (backward looking), devido a mecanismos de indexação e de contratos justapostos.
} 
onde:

$$
\begin{gathered}
k^{*}=\frac{a_{2} c_{0}-a_{0} c_{1}}{a_{2}+a_{1} c_{1}}, \quad \alpha=\frac{c_{1}}{a_{2}+a_{1} c_{1}} \\
\beta=\frac{a_{2} c_{1}}{a_{2}+a_{1} c_{1}}, \quad \gamma=\frac{b_{2}}{a_{2}+a_{1} c_{1}}
\end{gathered}
$$

Introduzindo-se o hiato do produto $h=y-\bar{y}$, o modelo anterior transforma-se em:

$$
\left\{\begin{array}{l}
h=k+\alpha(m-p)+\beta \pi^{e}+\gamma f \\
\pi=\pi^{e}+\delta h \\
\dot{\pi}^{e}=\theta\left(\pi-\pi^{c}\right)
\end{array}\right.
$$

A equação do mecanismo de expectativa adaptativa pode ser escrita com auxílio do operador definido por $D x=d x / d t$, do seguinte modo:

$$
\pi^{e}=\frac{\theta}{\theta+D} \pi
$$

Substituindo-se este valor de $\pi^{e}$ nas equações (27) e (28) obtćmse: ${ }^{7}$

$$
\left\{\begin{array}{l}
\dot{\pi}=-\delta \alpha \pi+\theta \delta(1+\beta \delta) h+\delta \alpha \mu \\
\dot{h}=-\alpha \pi+\theta \beta \delta h+\alpha \mu
\end{array}\right.
$$

onde admitimos que $f$ é constante e o símbolo $\mu$ é a taxa de crescimento da oferta de moeda, definida por:

$$
\mu=D m=\frac{d m}{d t}
$$

${ }^{7}$ Das equaçōes (28) e (29) segue-se que:

$$
\pi-\pi^{e}=\delta h \mathrm{e} \dot{\pi}^{e}=\theta\left(\pi-\pi^{e}\right)=\theta \delta h
$$

Derivando-se com relação ao tempo a equação $\pi=\pi^{e}+\delta h$, obtém-se: $\dot{\pi}=\dot{\pi}^{e}+\delta \dot{h}$, que é igual a $\dot{\pi}=\theta \delta h+\delta \dot{h}$, pois $\dot{\pi}^{e}=\theta \delta h$. Derivando-se com relação ao tempo a equação (27), obtém-se a equação (31): $\dot{h}=\alpha(\mu-\pi)+\beta \dot{\pi}^{e}=\alpha(\mu-\pi)+\beta \theta \delta h$, admitindo-se que $\dot{k}=\dot{f}=0$. Substituindo-se $\dot{h}$ na equação de $\dot{\pi}$ chegarse à equação (30). 
A matriz $A$ da expressão (5) neste caso é dada por:

$$
A=\left[\begin{array}{ll}
a_{11} & a_{12} \\
a_{21} & a_{22}
\end{array}\right]=\left[\begin{array}{cc}
-\delta \alpha & \theta \delta(1+\beta \delta) \\
-\alpha & \theta \beta \delta
\end{array}\right]
$$

cujo traço é igual a $-\delta(\alpha-\beta \theta)$, e cujo determinante é $\alpha \theta \delta$. Logo, para que o modelo seja estável devemos ter $\alpha>\beta \theta$.

A matriz $D$ neste exemplo é igual a:

$$
D=\frac{1}{\Phi_{2} \delta^{2}+\Phi_{1}}\left[\begin{array}{cc}
\Phi_{2} \delta^{2}(\rho+\theta) & \Phi_{1} \delta(\rho+\theta) \\
\Phi_{2} \delta(\rho+\theta) & \Phi_{1} \rho-\Phi_{2} \theta \delta^{2}
\end{array}\right]
$$

O traço desta matriz e seu determinante são:

$$
\begin{aligned}
& \operatorname{tr} D=\rho>0 \\
& |D|=-\frac{\Phi_{2} \theta \delta^{2}(\rho+\theta)}{\Phi_{2} \delta^{2}+\Phi_{1}}<0
\end{aligned}
$$

Admitiremos que antes do início do programa a economia estava em pleno emprego e que a moeda vinha crescendo a uma taxa constante e igual a $\mu^{*}$. Num modelo com expectativas adaptativas, a taxa de inflação esperada é igual à taxa de expansão monetária que vinha ocorrendo até então, isto é:

$$
\pi^{*}=\pi^{i}(0)=\pi^{e}(0)=\mu^{*}
$$

As constantes $C_{1}$ e $C_{2}$ das equações (25) e (26) devem satisfazer as equações (7) e (8), isto é:

$$
\left\{\begin{array}{l}
C_{1}=\mu^{*}+\delta C_{2} \\
-q C_{1}=-\delta q C_{2}+\theta \delta C_{2}
\end{array}\right.
$$

Logo:

$$
\begin{aligned}
C_{1} & =\left(1-\frac{q}{\theta}\right) \mu^{*} \\
C_{2} & =\frac{q \mu^{*}}{\delta \theta}
\end{aligned}
$$


As trajetórias da taxa de inflação $\pi$ e do hiato do produto $h$ são, então, dados por:

$$
\begin{aligned}
& \pi=\left(1-\frac{q}{\theta}\right) \mu^{*} e^{-q t} \\
& h=-\frac{q \mu^{*}}{\delta \theta} e^{-q t}
\end{aligned}
$$

A taxa de crescimento da oferta de moeda pode ser facilmente obtida com auxílio da equação (31), pois:

$$
\mu=\frac{1}{\alpha}(\dot{h}+\alpha \pi-\theta \beta \delta h)
$$

Substituindo-se os valores anteriores de $\pi$ e $h$ nesta expressão, obtém-se:

$$
\mu=\left(1-\frac{\alpha-\beta \theta}{\alpha \theta} q+\frac{q^{2}}{\alpha \theta \delta}\right) \mu^{*} e^{-q t}
$$

Observe-se que no momento inicial $(t=0)$, a taxa de expansão monetária ótima é diferente da taxa que vinha sendo mantida no passado $\left(\mu(0) \neq \mu^{*}\right)$. Por outro lado, há uma queda instantânea do produto, pois:

$$
h(0)=\frac{q \mu^{*}}{\delta \theta}<0
$$

A Figura 4 descrcve a trajetória da recessão criada pclo programa de cstabilização. Esta recessão ocorre porque durante todo o programa, a cada instante, a taxa de inflação observada é menor do que a taxa de inflação antecipada pelos agentes econômicos como indicado na Figura 5.

Seja $h\left(0_{-}\right)$o valor do hiato do produto antes de começar o programa de estabilização. Segue-se da equação (27) que:

$$
\begin{aligned}
& h(0)-h(0-)=\alpha[m(0)-m(0-)-p(0)-p(0-)]+ \\
& \quad \beta\left[\pi^{e}(0)-\pi^{e}(0-) 1+\gamma[f(0)-f(0-)]\right.
\end{aligned}
$$

Neste modelo não ocorrem por hipótese, saltos instantâneos na taxa de inflação esperada e no nível de preços, logo:

$$
\begin{aligned}
\pi^{e}(0) & =\pi^{e}(0-) \\
p(0) & =p(0-)
\end{aligned}
$$




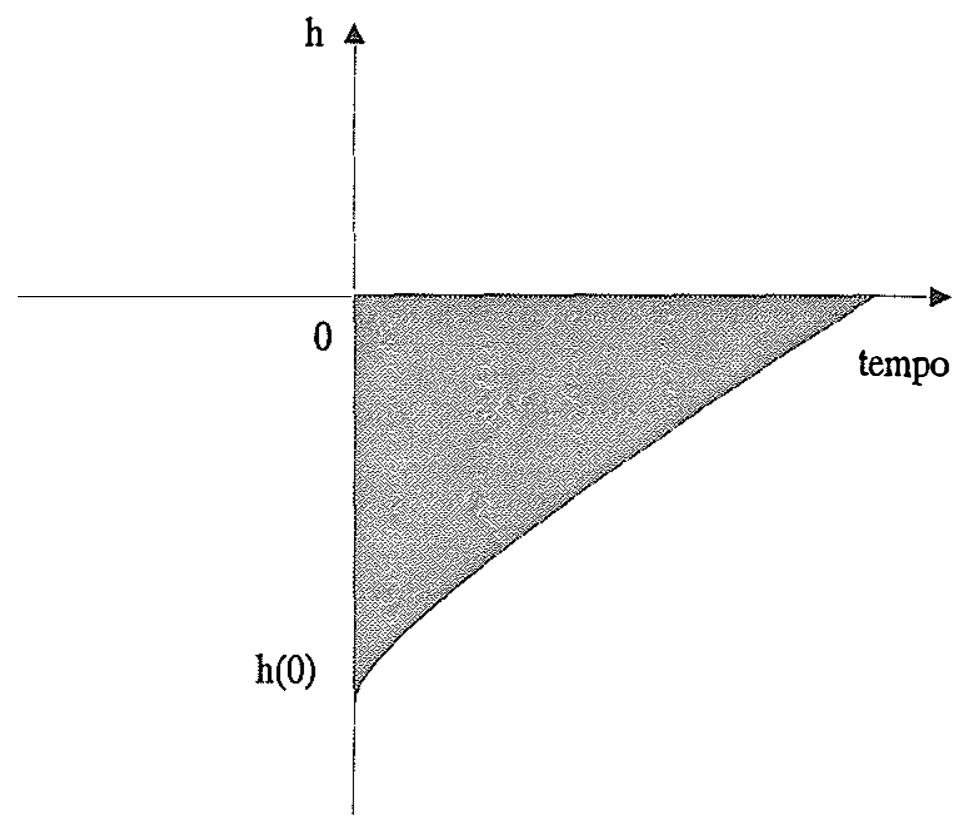

Figura 4.

Trajetória da recessão

Segue-se, portanto, que:

$$
h(0)-h(0-)=\alpha[m(0)-m(0-)]+\gamma[f(0)-f(0-)]
$$

Suponha-se que a política fiscal não seja alterada, $f(0)=f(0-)$, e que o hiato do produto antes de começar o programa de estabilização era igual a zero. Nestas circunstâncias:

$$
m(0)=m(0-)+\frac{1}{\alpha} h(0)
$$

Conclui-se, portanto, que a política monetária ótima do programa de estabilização começa com uma diminuição do estoque nominal de moeda, e que daí por diante a taxa de crescimento do estoque 


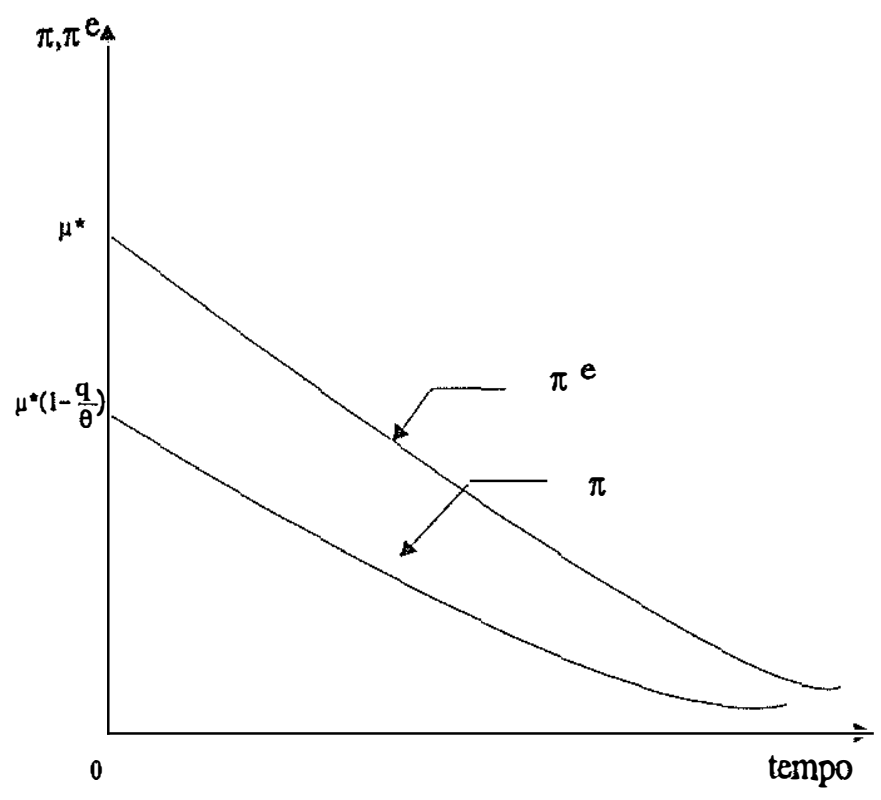

Figura 5.

Taxas de inflação atual e esperada

mais do seu nível de longo prazo, de acordo com a trajetória da Figura 7.

A política monetária ótima neste exemplo consiste: i) num choque monetário, no instante inicial, com a redução do estoque de moeda e ii) na redução gradual da taxa de crescimento do estoque de moeda ao longo do tempo. A economia muda do ponto $A$ para o ponto $B$ no diagrama de fases da Figura 3 , com a taxa de inflação cedendo um pouco e a economia entrando em recessão com o hiato do produto negativo. A redução da taxa de inflação e da recessão se fará gradualmente com a economia convergindo para o pleno emprego e para a inflação zero.

3.2. Ajuste instantâneo no mercado de ativos e inércia nos preços.

Considere, agora, o modelo formado pelas seguintes equações: 


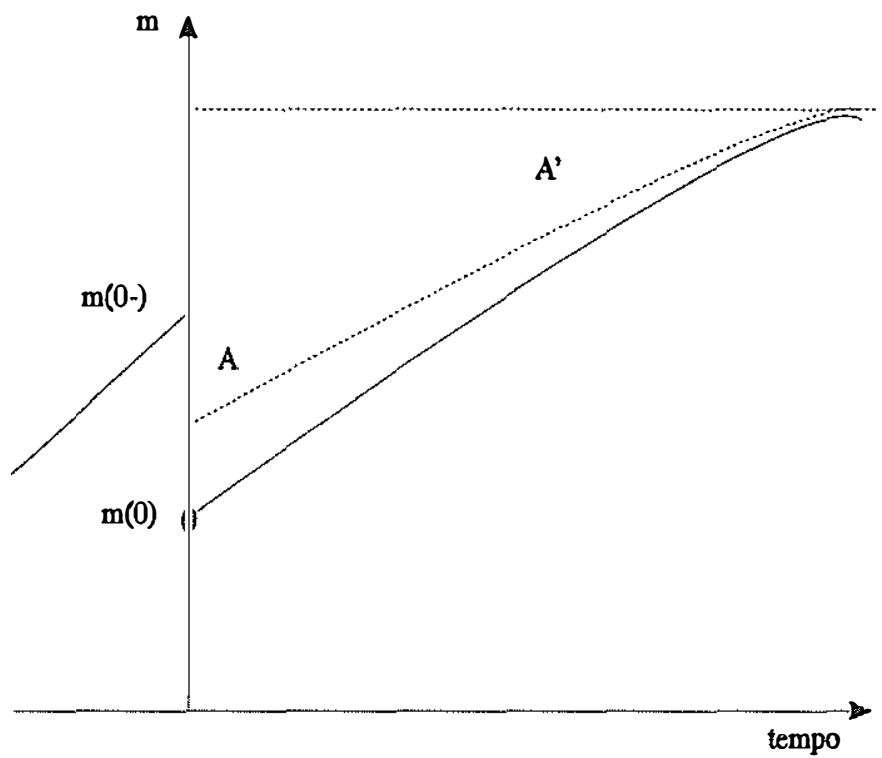

Figura 6.

A trajetória da política monetária ótima

$$
\left\{\begin{array}{l}
h=k+\alpha(m-p)+\beta \pi+\gamma f \\
\pi=\pi^{e}+\delta h \\
\dot{\pi}^{e}=\theta\left(\pi-\pi^{e}\right)
\end{array}\right.
$$

A equação (36) resulta da equação (27) quando se faz $\pi^{e}=\pi$. Esta hipótese equivale a dizer que a previsão é perfeita no mercado de ativos, pois a taxa de juros nominal é igual à soma da taxa de juros real com a taxa de inflação observada. No lado da oferta agregada, existe um componente inercial na taxa de inflação, que é representado pelo mecanismo de expectativa adaptativa: As equações (37) e (38) são idênticas às equações (28) e (29).

Diferenciando-se com relação ao tempo ambos os lados da equação (36) e substituindo-se (38) em (37), obtém-se o seguinte sistema 
Política monetária ótima

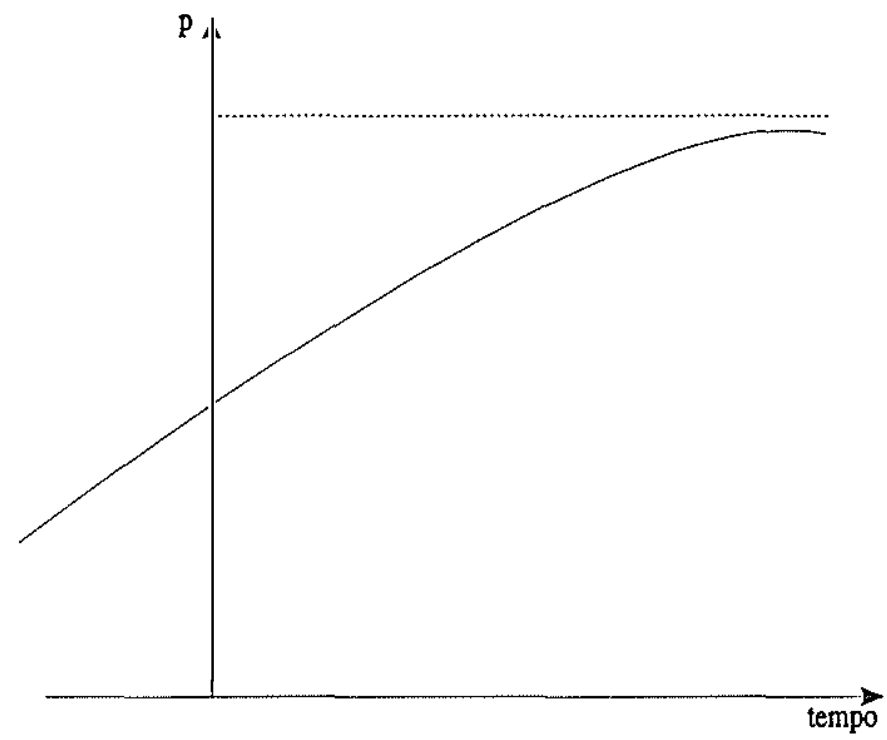

Figura 7.

A trajetória do índice de preços

de equações:

$$
\left\{\begin{array}{l}
\dot{h}=\alpha(\mu-\pi)+\beta \dot{\pi} \\
\dot{\pi}=\delta \beta h+\delta \dot{h}
\end{array}\right.
$$

que pode ser escrita como:

$$
\left[\begin{array}{l}
\dot{\pi} \\
\dot{h}
\end{array}\right]=\frac{1}{1-\beta \delta}\left[\begin{array}{cc}
-\delta \alpha & \delta \theta \\
-\alpha & \beta \delta \theta
\end{array}\right]\left[\begin{array}{l}
\pi \\
h
\end{array}\right]+\frac{1}{1-\beta \delta}\left[\begin{array}{c}
\alpha \delta \mu \\
\alpha \mu
\end{array}\right]
$$

A matriz $A$ neste exemplo é dada, então, por:

$$
A=\frac{1}{1-\beta \delta}\left[\begin{array}{cc}
-\delta \alpha & \delta \theta \\
-\alpha & \beta \delta \theta
\end{array}\right]
$$


O traço e o determinante desta matriz são iguais a:

$$
\begin{aligned}
& \operatorname{tr} A=\frac{-\delta(\alpha-\beta \theta)}{1-\beta \delta} \\
& |A|=\frac{\alpha \delta \theta}{1-\beta \delta}
\end{aligned}
$$

Para que este modelo seja estável, seus parâmetros devem satisfazer a seguinte desigualdade:

$$
\beta<\min \left\{\frac{\alpha}{\theta}, \frac{1}{\delta}\right\}
$$

O traço e o determinante da matriz $D$, da qual é obtida a solução ótima da política monetária, são dados por:

$$
\begin{aligned}
& \operatorname{tr} D=\rho \\
& |D|=-\frac{\Phi_{2} \theta \delta^{2}(\rho+\theta)}{\delta^{2} \Phi_{2}+\Phi_{1}}
\end{aligned}
$$

Observe-se que o determinante da matriz $D$ neste caso é exatamente igual ao valor do exemplo anterior. As trajetórias da taxa de inflação e do hiato do produto são, então, as mesmas das equações (32) e (33), repetidas aqui por conveniência:

$$
\begin{aligned}
& \pi=\left(1-\frac{q}{\theta}\right) \mu^{*} e^{-q t} \\
& h=-\frac{q \mu^{*}}{\delta \theta} e^{-q t}
\end{aligned}
$$

A taxa de crescimento do estoque de moeda é obtida a partir da equação (38), ou seja:

$$
\mu=\pi+\frac{1}{\alpha}(\dot{h}-\beta \dot{\pi})
$$

Substituindo-se os valores de $\pi$ e $h$ nesta expressão, obtém-se:

$$
\mu=\left[1-\frac{(\alpha-\beta \theta)}{\alpha \theta} q+\frac{(1-\beta \delta)}{\alpha \theta \delta} q^{2}\right] \mu^{*} e^{-q t}
$$


Comparando-se a taxa de crescimento do estoque de moeda, no momento inicial, neste caso com aquela da fórmula (34) é fácil verificar-se que a taxa de crescimento do estoque de moeda agora é menor do que a taxa correspondente do exemplo anterior.

Para analisar o que acontece com o estoque de moeda no momento inicial do programa de estabilização, utilizamos a equação (36) para escrever:

$$
\begin{aligned}
h(0)-h(0-)=\alpha[m(0)- & m(0-)-p(0)+p(0-)] \\
& +\beta[\pi(0)-\pi(0-)]+\gamma[f(0)-f(0-)]
\end{aligned}
$$

Adotando-se as mesmas hipóteses do exemplo precedente $[h(0-)$ $=0, p(0)=p(0-), f(0)=f(0-)]$ e o fato de que:

$$
\pi(0)-\pi(0-)=\left(1-\frac{q}{\theta}\right) \mu^{*}-\mu^{*}=-\frac{q \mu^{*}}{\theta}
$$

O estoque nominal de moeda é igual a:

$$
m(0)=m(0-)+\frac{1}{\alpha} h(0)+\frac{\beta q \mu^{*}}{\alpha}
$$

Este estoque inicial de moeda é maior do que o estoque correspondente do exemplo anterior, como se pode verificar comparando-se as expressões (42) e (35). A trajetória $A A^{\prime}$ da Figura 6 mostra a política monetária ótima neste exemplo: uma redução do estoque nominal de moeda no momento inicial do programa de estabilização, seguido por uma monetização à taxa decrescente do estoque de moeda.

A diferença básica entre este exemplo e o anterior deve-se ao comportamento da taxa de juros nominal. Como ela responde imediatamente ao declínio da taxa de inflação, a demanda de moeda aumenta. Conseqüentemente, o estoque de moeda inicial deve ser maior do que quando tal fato não acontece.

\section{Conclusão.}

Existe uma longa discussão na literatura econômica quanto aos benefícios e custos associados aos tratamentos gradualista e de choque 
no combate à inflação. Recentemente, na América Latina, foi aplicado tratamento de choque no controle dos preços dos bens e serviços na economia. Todavia, nas áreas fiscal e monetária, a opção dos encarregados pela política econômica foi pela omissão e negligência, ou quando muito se declarou intenções de ajustes fiscais, que não foram executados na prática. A inflação ignorou estes programas de estabilização e continuou sua trajetória explosiva.

Economistas a favor de um tratamento de choque aconselham o congelamento do estoque de moeda como uma maneira eficaz de combate à inflação. Economistas a favor de um tratamento gradualista, como Friedman, afirmam que "an ideal policy would... involve an initial decline in monetary growth, a subsequent rise when declining inflation reduces velocity, and a final decline to the desired long-run level when velocity stabilizes" (Friedman 1985, p.19).

A determinação da política monetária ótima desenvolvida neste trabalho, num modelo de uma economia em que a inflação tem um componente inercial, mostra que a política monetária ótima requer que no início do programa de estabilização o estoque de moeda deve ser diminuído, como propõe o tratamento do choque, e que, subseqüentemente, a taxa de crescimento do estoque de moeda deve ser reduzida gradualmente ao longo do tempo, como propõe o tratamento gradualista da inflação.

(Submetido em outubro 1991. Revisado em fevereiro 1992)

\section{Referências}

Beavis, B. \& Dobbs I. 1990. Optimization and Stability Theory for Economic Analysis. Cambridge: Cambridge University Press.

Buiter, W.H. \& Miller M.H. 1985. "Costs and Benefits of an Anti-Inflationary Policy: Questions and Issues." In Argy, V. \& Nevile, orgs., Inflation and Unemployment: Theory, Experience and Policy Making. Londres: George Allen \& Unwin.

Clark, C.W. 1976. Mathematical Bioeconomics: The Optimal Management of Able Resources. New York: John Wiley.

Dornbusch, R. 1976. "Expectations and exchange rate dynamics." Journal of Political Economy 84: 1161-1176. 
Friedman, M. 1985. "Monetarism in rhetoric and in practice." In Ando, A., Eguchi, H., Farmer, R. \& Suzuki, Y. org., Monetary Policy in Our Times. Cambridge: MIT Press.

Kamien, M.I. \& Schwartz, N.L. 1981. "Dynamic optimization: the calculus of variations and optimal control." In Economics and Management. New York: North-Holland.

Kendrick, D.A. 1976. "Applications of control theory to macroeconomics." Annals of Economic and Social Measurement 5:171190.

Pitchford, J.D. \& Turnovsky S.J. 1977. Applications of Control Theory to Economic Analysis. Amsterdam: North-Holland.

Seierstad, A. \& Sydsaeter K. 1987. Optimal Control Theory with Economic Applications. Amsterdam: North-Holland.

Theil, H. 1964. Optimal Decisions Rules for Government and Industry. Amsterdam: North-Holland.

Tu, P. N.V. 1984. Introductory Optimization Dynamics. Berlin: Springer-Verlag.

Turnovsky, S. 1981. "The optimal intertemporal choice of inflation and unemployment: an analysis of the steady state and transitional dynamics." Journal of Economic Dynamics and Control 3: $357-384$. 